\title{
Normal Pressure Hydrocephalus Presentation with a Large Pseudomeningocele
}

\author{
Ehsan Alimohammadi ${ }^{1 *}$, Guive Sharifi ${ }^{2}$, Mohammad Samadian ${ }^{2}$, Kaveh Ebrahimzadeh ${ }^{3}$, \\ Omidvar Rezaei ${ }^{4}$
}

\author{
${ }^{1} \mathrm{MD}$, Resident of Neurosurgery, Shahid Beheshti University of Medical Sciences, Tehran, Iran \\ ${ }^{2}$ MD, Associate Professor of Neurosurgery, Shahid Beheshti University of Medical Sciences, Tehran, Iran \\ ${ }^{3} \mathrm{MD}$, Assistant Professor of Neurosurgery, Shahid Beheshti University of Medical Sciences, Tehran, Iran \\ ${ }^{4} \mathrm{MD}$, Professor of Neurosurgery, Shahid Beheshti University of Medical Sciences, Tehran, Iran
}

*Corresponding Author Address: Loghman Hakim Hospital, Makhsoos Street, South Karegar Avenue, Tehran, Iran. Tel: +98-9181262143. Fax: +98-21-55417547. Email: hafez125@gmail.com

Article Type: Case Report

Received: March 31, 2017, Last Revised: April 1, 2017, Accepted: April 1, 2017, Published: September 28, 2017

\section{Abstract}

Background and Importance: This interesting case is about the presence of normal pressure hydrocephalus and a large pseudomeningocele at the same time after 13 months of posterior fossa surgery. Although the occurrence of a pseudomeningocele following posterior fossa surgery is not so rare, such a late large pseudomeningocele development with signs and symptoms of NPH after 13 months of surgery is rather peculiar.

Case Presentation: A 59-year-old man referred to our clinic for a cerebellopontine angle tumor. The patient presented with right hearing loss and mild facial palsy. We operated the patient with retrosigmoid approach, and we discharged him after five days. After about 13 months, the patient referred to our center again with complaint of progressive bulging of previous surgical region and gait apraxia, urinary incontinence and dementia. A large pseudomeningocele in the site of previous surgery was seen.

Conclusion: The authors presented a case report on late development (13 months) of a huge pseudomeningocele following vestibular schwannoma surgery with signs and symptoms of normal pressure hydrocephalus. Such a late big pseudomeningocele presentation with signs and symptoms of normal pressure hydrocephalus after posterior fossa surgery is peculiar.

Keywords: Hydrocephalus; Pseudomeningocele; Intracranial; Pressure

Please cite this paper as: Alimohammadi E, Sharifi G, Samadian M, Ebrahimzadeh K, Rezaei O. Normal Pressure Hydrocephalus Presentation with a Large Pseudomeningocele. Iran J Neurosurg. 2017;3(2):73-77.

\section{Background and Importance}

We presented a case with normal pressure hydrocephalus (NPH) and a large pseudomeningocele at the same time after 13 months of posterior fossa surgery. A pseudomeningocele may develop after posterior fossa surgery, but the presence of such a late large pseudomeningocele with signs and symptoms of NPH after 13 months of surgery is rather peculiar.

\section{Case Presentation}

A 59-year-old man referred to our clinic with a cerebellopontine angle (CPA) tumor. On admission, the patient gave informed written consent to participate in the study. He presented with right-side hearing loss and mild facial palsy. Brain magnetic resonance imaging (MRI) showed a large right CPA
(Figure 1). We operated the patient with retrosigmoid approach, and the pathology revealed a schwannoma. The patient was discharged with good general condition five days after operation. Thirteen months later, the patient referred again complaining of progressive bulging of previous surgical field from about two months before that, and gait apraxia, urinary incontinence and dementia from about 20 days before that.

Neurological examination revealed Mini-Mental State Examination (MMSE) score 23/30. Motor system examination showed normal tone and power in all four limbs. Deep tendon jerks were normal. Gait assessment revealed wide-based stance. His steps were slow, short, and shuffling. His feet could not be raised much above the ground as if they were glued but his visual 


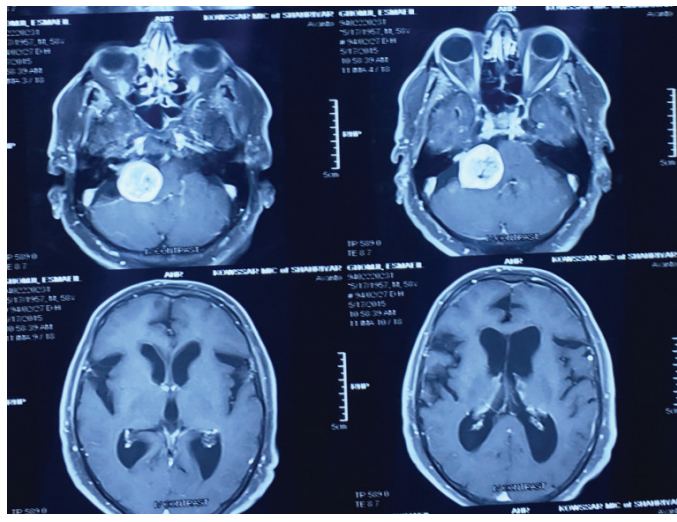

Figure 1. Initial Patient Brain MRI (Right CPA Tumor)

field and acuity were normal. There was no papilledema. A large pseudomeningocele in the site of previous surgery was seen (Figure 2).

Considering this information, the patient was investigated. His hematological and biochemical parameters were normal. Brain imaging showed fluid collection in the region of the right parietal-occipital scalp and symmetric dilatation of all the ventricles without periventricular edema. There were no significant white matter abnormalities. These findings were seen on T1, T2 weighted and T2 fluid-attenuated inversion recovery (FLAIR) images (Figure 3). Finally, NPH was diagnosed, and the cerebrospinal fluid (CSF) tap test (TT) was done; 50 milliliters (ml) of CSF was drained. The opening pressure was 180 millimeters $(\mathrm{mm})$ of water, and cyto-biochemical examination of CSF showed proteins $21 \mathrm{mg} / \mathrm{dl}$, sugar $70 \mathrm{mg} / \mathrm{dl}$, and cells 0 . Pre- and post-test assessments of gait and cognitive functions were performed which revealed remarkable improvement in patient's gait. With such a significant improvement with CSF TT, the patient underwent ventriculoperitoneal shunt surgery and afterward there was almost complete recovery of both cognitive and gait disturbance. The patient was discharged at forth postoperative day. After three weeks, he was re-examined, and the shunt was performing well. Neurological examination revealed MMSE score as $28 / 30$, and he had stable improved cognitive and gait status. The pseudomeningocele disappeared thoroughly (Figures 4,5). He is still under follow-up to search shunt functions and its associated complications.

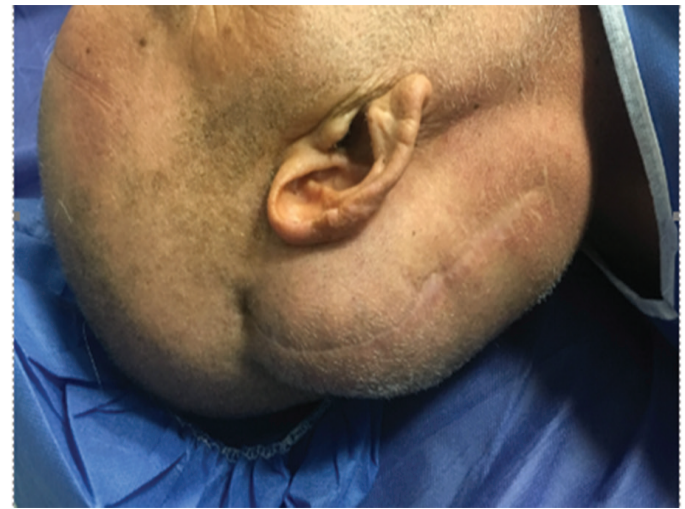

Figure 2. 13 Months After Surgery. The Patient Presented with Progressive Bulging of Surgical Field.

\section{Discussion}

A pseudomeningocele is an abnormal body of cerebrospinal fluid communicating with the CSF space in which the fluid has no membrane around, yet it exists in a cavity of soft tissues [1]. However, the pathophysiology of pseudomeningocele raises ambiguities for some researchers and scientists who consider the potential causes of pseudomeningocele in the subarachnoid space such as intradural defects. On the contrary, absorbing CSF along with other problems including subarachnoid scarring and hydrocephalus may also play a vital role in some others' opinion [2]. The time of pseudomeningocele occurrence was reported to range from 5 to 115 days after surgery (mean: 18.2 days) [3].

The symptoms constellation of NPH include gait apraxia, urinary disturbance and memory impairment. According to reports, gait disturbance is the most common symptom occurring earlier than other complications [4]. Much more research needs to be performed to discover the aspects of pathophysiology of NPH which is assumed to be related to decreased absorption of cerebrospinal fluid rather than over-production [5]. There are two types of NPH: Idiopathic and secondary. The latter may result from brain trauma and tumors, subarachnoid hemorrhage, infection of the central nervous system, or occur after cranial surgery [6]. Relkin and his colleagues in their study suggested that NPH had a slow onset in someone over 40 years old with worsening symptoms occurring for three to six months without other neurological presentations. Brain imaging should show 

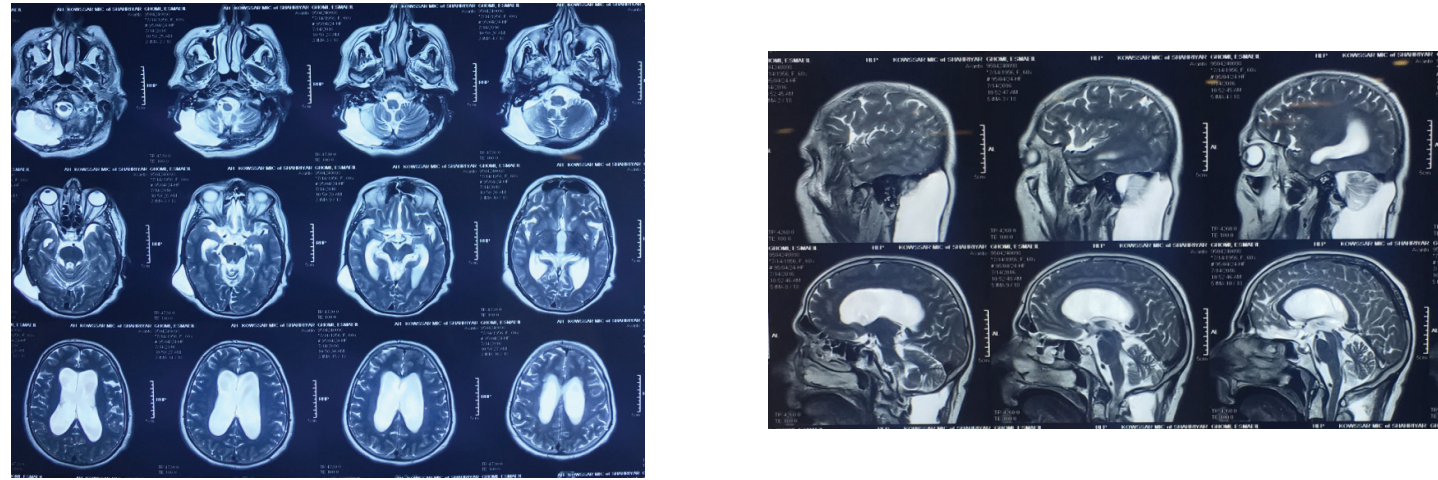

Figure 3. Brain MRI of the Patient Demonestrating a Large Pseudomeningocele in Previous Surgical Field, Ventriculomegaly Without Periventricular Edema.

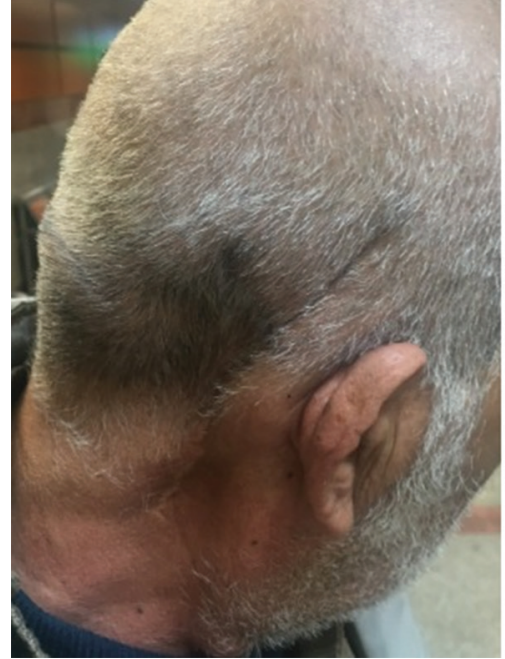

Figure 4. Three Weeks After VP Shunting. The Pseudomeningocele Was Resolved Completely.

ventriculomegaly without periventricular white matter change and evidence of parenchymal cerebral atrophy. For a strong diagnosis, a patient should have the gait disturbance and at least two of nine following symptoms: decreased step height and length, a wide-based gait, slowed walking, truncal swaying during walking, toes deviating outward, spontaneous retropulsion, difficulty turning, and difficulty keeping balance while walking [7-10]. According to references, there are very important factors related to intracranial pressure (ICP) namely the volume of intracranial fluid, the elastance, the contribution of the atmosphere, and the orientation of the craniospinal axis relative to the gravitational vector [11-13].

According to Tanna and colleagues' study, cranial CSF volume is about $164.5 \mathrm{~mL}$, with a range of 62.2 to $267 \mathrm{~mL}$. Ventricular

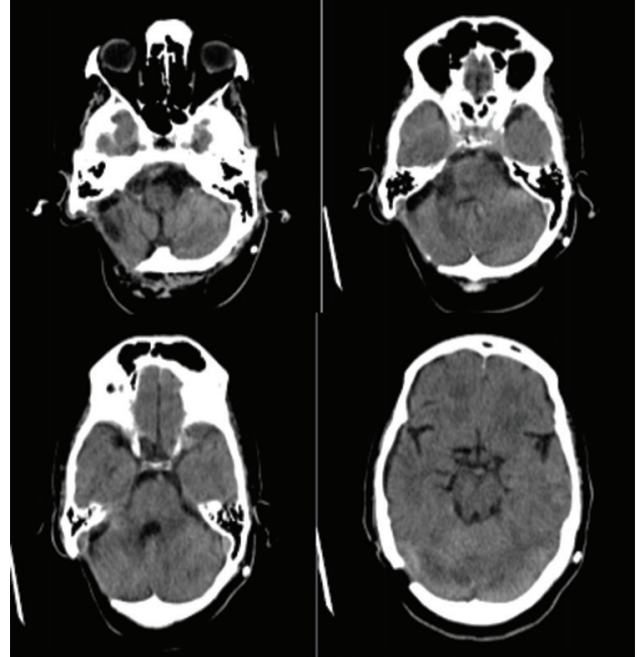

Figure 5. The Pseudomeningocele Was Resolved Completely Three Weeks After VP Shunting, Shown in MRI.

volume also varies considerably from 7.49 to $70.5 \mathrm{~mL}$, with a mean of $31.9 \mathrm{~mL}$ [14]. Elastance is defined by the pressure change per unit of volume change. We cannot consider this relationship here absolutely linear in all volumes with the same status in all physiologic conditions. Compliance is considered as the opposite of elastance. Two main ways of compensation include expansion or loss of volume when volume is added to the system. In a physiologic sense, this can occur either by distention of the spinal dura mater or by displacement of CSF and blood $[13,15,16]$.

According to prior studies and references, there was linear relationship between intracranial volume and ICP which can be better understood by showing the response of ICP to volume added into the neural axis (Figure 6.A). Ryder et al. 

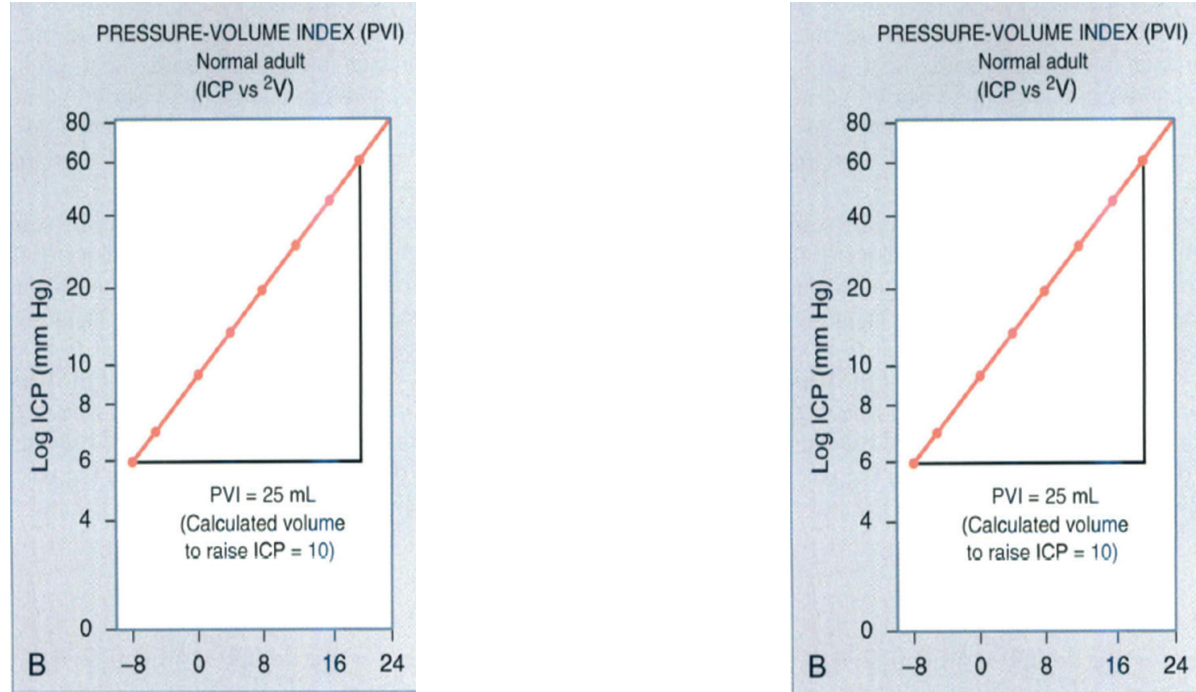

Figure 6. (A) Relationship between Intracranial Volume and ICP (B) Relationship between Intracranial Volume and Logarithm of ICP

explained that this relationship described hyperbolic curvein normaladults. According to prior studies, in spatial compensation as volume augments the intracranial pressure is minimally influenced. Still, the increased pressure changes per unit volume occurred with declining compliance; this portion is named the spatial decompensation [13,15-17]. As explained in the exemplified curve with compliance diminishing, the slope had a swift growth during spatial decompensation [13,17-19]. According to references, placing intracranial pressure logarithmically against volume is an alternative method, which gives a straight line. Its slope is the pressure-volume index (PVI), or the calculated volume in milliliters needed to raise ICP by a factor of 10 (Figure 6.B). In normal adults, the index ranges from 25 to $30 \mathrm{~mL}$. Then compliance decreases through pathologic processes (decreased index). Finally, a few changes volume would cause significant alterations in pressure $[13,15,17]$.

According to above, we suggest a hypothesis that could explain this case. The pseudomeningocele and the hydrocephalus result from the same hydrodynamic mechanisms. Progressive hydrocephalus can induce both ventricular and extradural collection dilatation. It seems that the surgical procedure to remove the vestibular schwannoma weakens the dural layer creating a "locus minori resistentiae" through which the CSF finds its way to the soft tissues at the base of the neck, forming the pseudomeningocele. We assume that the initial problem was a chronic hydrocephalus but the important point in this patient was the absence of intracranial hypertension signs and symptoms including papilledema, periventricular edema, and high opening pressure in tap test. It seems that the large pseudomeningocele acted as a reservoir to increase the system compliance, therefore prolongation of spatial compensation phase, and as a result, manifestation of a chronic hydrocephalus in delayed mode as NPH after 13 months of surgery without signs and symptoms of elevated ICP occurred. Unfortunately the patient did not refer to us for follow-up visits. More images in followup period could be helpful in understanding how the hydrocephalus developed over time.

\section{Conclusion}

The authors of this study presented a case report on late development (13 months) of a huge pseudomeningocele following vestibular schwannoma surgery with symptoms of NPH. Such a late big pseudomeningocele presentation with signs and symptoms of NPH after posterior fossa surgery can be considered peculiar.

\section{Funding}

This research did not receive any specific grant from funding agencies in the public, commercial, or not-for-profit sectors. 


\section{Conflicts of interest}

No potential conflicts of interest were disclosed.

\section{Author's Contribution}

Conception and Design: Guive Sharifi, Ehsan Alimohammadi

Data Collection: Ehsan Alimohammadi, Kaveh Ebrahimzadeh

Drafting the Article: Mohammad Samadian Critically Revising the Article: Omidvar Rezaei Reviewed Submitted Version of the Manuscript: Guive Sharifi

Approved the Final Version of the Manuscript: Omidvar Rezaei

\section{References}

1.Kapusuz Z, Ozkiris M, Okur A, Saydam L. Pseudomeningocele presenting as a cyst of the external auditory canal. J Craniofac Surg. 2013;24:235-7.

2.Saigal, Rajiv, et al. "Rotational Pericranial Flap for Repair of Refractory Posterior Fossa Pseudomeningocele." Cureus 6.1 (2014).

3.Mehendale NH, Samy RN, Roland PS. Management of pseudomeningocele following neurotologic procedures. Otolaryngol Head Neck Surg. 2004;131:253-62.

4.Hebb, Adam O., and Michael D. Cusimano. "Idiopathic normal pressure hydrocephalus: a systematic review of diagnosis and outcome." Neurosurgery 49.5 (2001): 1166 1186

5.Bergsneider, Marvin, et al. "Surgical management of idiopathic normal-pressure hydrocephalus." Neurosurgery 57.3 (2005): S2-29.

6.Vanneste, Jan, et al. "Shunting normal pressure hydrocephalus: the predictive value of combined clinical and CT data." Journal of Neurology, Neurosurgery \& Psychiatry 56.3 (1993): 251-256.

7.Relkin N, Marmarou A, Klinge P, et al. Diagnosing idiopathic normalpressure hydrocephalus. Neurosurgery. 2005;57(3 Suppl):S4-16.

8.Vanneste JA. Three decades of normal pressure hydrocephalus: are we wiser now? [Editorial]. J Neurol Neurosurg Psychiatry 1994;57:1021-25.

9.Wikkelso C, Andersson H, Blomstrand C, et al. Normal pressure hydrocephalus. Predictive value of the cerebrospinal fluid tap-test. Acta Neurol Scand. 1986;73:566-73.

10.SÃCELEANU, VICENDIU, DANIELA RADU, and ADRIANA SÃCELEANU. "NORMAL-PRESSURE HYDROCEPHALUS, CASE REPORT" Acta Medica Transilvanica (2014). 19:2.

11.Walchenbach R, Geiger E, Thomeer RT, et al. The value of temporary external lumbar CSF drainage in predicting the outcome of shunting on normal pressure hydrocephalus. J Neurol Neurosurg Psychiatry. 2002;72:503-6.

12.Verrees M, Selman WR. Management of normal pressure hydrocephalus. Am Fam Physician. 2004;70:1071-8.

13.Winn R, Youmans neurological surgery 6th ed. Philadelphia, PA, 2011

14.Tanna, Nitin K., et al. "Analysis of brain and cerebrospinal fluid volumes with MR imaging: impact on PET data correction for atrophy. Part II. Aging and Alzheimer dementia." Radiology 178.1 (1991): 123-130.

15.Shapiro, K., A. Marmarou, and K. Shulman. "Characterization of clinical CSF dynamics and neural axis compliance using the pressure-volume index: I. The normal pressure-volume index." Annals of neurology 7.6 (1980): 508-514
16.Wagshul, Mark E., et al. "Amplitude and phase of cerebrospinal fluid pulsations: experimental studies and review of the literature." Journal of neurosurgery 104.5 (2006): 810-819.

17.Marmarou, Anthony, et al. "Development of guidelines for idiopathic normal-pressure hydrocephalus: introduction." Neurosurgery 57.3 (2005): S2-1.

18.Hakim, C. A., R. Hakim, and S. Hakim. "Normalpressure hydrocephalus." Neurosurgery clinics of North America 12.4 (2001): 761

19.Hebb, Adam O., and Michael D. Cusimano. "Idiopathic normal pressure hydrocephalus: a systematic review of diagnosis and outcome." Neurosurgery 49.5 (2001): 11661186 